\title{
Information and communication technology in obesity
}

\author{
Suset Dueñas ${ }^{1}$, María Arnoriaga ${ }^{2}$, Alberto Brizuela ${ }^{3}$, Nikhyl Jhangiani ${ }^{4}$, Ronald Gambino ${ }^{5}$, Kevin M Pantalone ${ }^{5}$, James B. Young ${ }^{5,6}$ and \\ Bartolome Burguera ${ }^{5,6 *}$
}

${ }^{1}$ Endocrinology Section, Hospital Virgen del Rocío, Sevilla, Spain

${ }^{2}$ Endocrinology Section, Hospital Gregorio Marañon, Madrid, Spain

${ }^{3}$ Department of Medicine. St. Barnabas. Bronx, New York

${ }^{4}$ Distance Health, Office of Clinical Transformation, Cleveland Clinic, Cleveland, USA

${ }^{5}$ Endocrinology \& Metabolism Institute, Cleveland Clinic, Cleveland, USA

${ }^{6}$ National Diabetes and Obesity Research Institute, Tradition, Mississippi, USA

Obesity is a chronic disease resulting from continuous positive energy balance. According to the Centers for Disease Control and Prevention (CDC) more than one-third of US adults have obesity [1]. In 2014, the World Health Organization (WHO) estimated that over 600 million individuals suffered obesity. Overall, about $13 \%$ of the world's adult population ( $11 \%$ of men and $15 \%$ of women) had obesity in 2014 [2]. If this pattern continues, it is estimated that 573 million individuals will suffer this chronic disease by 2030 [3]. Also more than 36 million die annually from non-communicable diseases, like cardiovascular diseases, cancers, chronic respiratory diseases and diabetes [4]; evidence has shown that obesity is associated with the development of all of these diseases. Accordingly, the prevention and treatment of obesity are necessarily urgent. While the massive growth of information and communication technology (ICT) has contributed to more sedentary lifestyles and weight gain, interestingly, these new technologies, inexpensive and portable nature, are becoming more and more useful in the fight against the obesity epidemic.

The purpose of this brief review is to evaluate the scientific evidence supporting the usefulness of information and communication technology in the management of obesity in clinical practice.

\section{Information and Communication Technology / E-Health}

The term electronic health "e-Health" did not start to be used in the literature until 1999, [5] and was originally defined as an emerging field at the intersection of medical informatics, public health, and business; basically, health services and information, delivered or enhanced through the Internet and related technologies.

Eysenbach [6] established 'The 10 e's in e-Health': efficiency, enhancing quality, evidence based, empowerment, encouragement, education, enabling, extending, ethics and equity [5]. For other authors, e-Health was considered as a large variety of medical informatics applications for facilitating the management and delivery of health care, including the spread of health-related information, storage and exchange of clinical data, inter-professional communication, computer-based support, patient-provider interaction, education, health service management, health communities, and telemedicine among other functions.

At the present time, e-Health characterizes a state-of-mind, a way of thinking, an attitude, and a commitment for networked, global thinking, to improve health care locally, regionally, and worldwide by using information and communication technology.
Telemedicine is considered a key element of the full context of e-Health [5] as defined by the American Telemedicine Association (ATA); however, when researchers describe their work, telemedicine is more specific than the generic term e-Health that is a common name for all health technological fields [7].

In the last few years, several e-Health elements have already been incorporated gradually into clinical practice, especially in developed countries, like clinical decision support through electronic information, electronic health records, computerized physician order entry, e-Prescribing, compliance with government regulations such as the Affordable Care Act and "Meaningful Use" among others. However, telemedicine is still far from its widespread use in routine practice.

On the other hand, due to the massive development in mobile communications using smart mobile devices that support $3 \mathrm{G}$ and $4 \mathrm{G}$ mobile networks for data transport, a new figure within e-Health, the " $\mathrm{m}$-Health", has been established as the new edge on healthcare innovation [8]. For this reason, particularly for the big growth of $\mathrm{m}$-Health applications, the concept of "self-management" through selfmonitoring healthcare devices is gaining more and more acceptance since 2005 [9]. Of importance is that $64 \%$ of American adults now own a smartphone of some kind, up from $35 \%$ in the spring of 2011. Also $\sim 60 \%$ of smartphone owners use their phone to look up information about a health condition [10].

\section{E-Health in obesity management}

The obesity epidemic [11] is the result of the combination of excessive caloric intake with a sedentary lifestyle over a long period. The guidelines for obesity management indicate that weight loss of $3-5 \%$ is likely to result in reduction of triglycerides, blood glucose and hemoglobin A1C levels; and reductions of $10 \%$ of total body weight will improve blood pressure, LDL cholesterol and increase HDL cholesterol, among other advantages [10]. Some clinical trials have shown that an intensive lifestyle modification is the gold -standard treatment for obesity. The Look AHEAD study showed that $50 \%$ of

Correspondence to: Barto Burguera, National Diabetes and Obesity Research Institute, Tradition, MS 39532, Endocrinology and Metabolic Institute. Cleveland Clinic, 9500 Euclid Ave. /M62| Cleveland, OH 44195, USA, Fax: 216445-1656; E-mail: BURGUEB@ccf.org

Key words: obesity, telemedicine, e-health, communication technology

Received: June 22, 2017; Accepted: July 19, 2017; Published: July 21, 2017 
patients included in the lifestyle intervention, maintained weight loss $\geq 5 \%$ at 8 years of follow up [12]. In the Diabetes Prevention Program (DPP) lifestyle interventions also succeeded in producing long-term (10-year) weight loss [13].

To achieve this type of weight loss in clinical practice, patients with obesity should be included in multidisciplinary programs with combined treatment options (diet, physical activity, cognitivebehavioral and pharmacological) [14]. This approach has suboptimal outcomes sometimes because of difficulties in access, costs, lack of staff and resources to improve the follow-up, treatment adherence, and long-term efficacy. Most patients regain weight in about one-third of the cases after the first year with starting these life style intervention programs [11]. Thus, the main challenge of treating obesity is, not to only accomplish weight loss, but to maintain long-term weight loss [15]. Several factors have been associated with weight loss maintenance; specifically, intensive lifestyle modifications $[12,13]$ with a prolonged extended treatment after the weight loss phase, have shown promising long-term weight loss outcomes [15]. The time commitment to attend the monthly appointments, could be overcome by leveraging mobile phones and virtual visits, to decrease the time commitment and missed time from work that patients may experience, which may improve compliance with the program. This may improve the longterm compliance that is currently a limiting factor with the office based approach to management. For all these circumstances, the application of e-Health in the prevention and treatment of overweight and obesity could be a great tool to help patients to maintain their weight loss.

Several authors have shown the usefulness of e-Health in the management of chronic conditions known to require long-term follow up, as well as self-management by the patient. Rosser et al. [16] investigated the use of technology in achieving behavior changes in chronic illness (depression, diabetes, cardiovascular disease, asthma, etc). They concluded that behavioral changes could be achieved by using technological innovations and that self-management systems could provide a practical method for understanding and monitoring these conditions, as well as therapeutic guidance to encourage healthy behaviors. They reported a lower dropout rate when the interventions included some online therapist involvement (supervision, assistance, or instruction).

Avery et al. [17], using a chronic disease management program (Healthcare Navigator, American Health Data Institute), described the positive impact that telephone based management coupled with motivational interviewing techniques and counselling performed by nurses, had in helping patients with chronic diseases like metabolic disorders to develop skills for disease self-management which could result in important health care savings.

In the case of obesity, the use of e-Health has been more limited, and the few interventions published have supported its usefulness in clinical practice. Two clinical trials for weight loss management $[12,13]$ indicate that the ideal therapies should include behavior modification therapy, self-monitoring of individual data with personalized recommendations and feedback [18]. Self-monitoring behaviors in weight management include weighing on a regular basis, frequent tracking of food, physical activity, and television or computer use [19]. These strategies have shown more adherence and effectiveness on weight control programs and most international chronic disease clinical guidelines recommend the application of self-management programs in clinical practice $[9,19,20]$.

\section{Technology based tools used in the weight management}

\section{Web based interventions}

Web interventions were one of the first e-Health technologies used for weight control in the context of research and as consumer products [21]. The spectrum of web-based interventions ranges from web platforms, specifically designed for a particular clinical trial to simply sending e-mails to improve patient's adherence to the intervention and their feedback. Kodama et al. [22] conducted a meta-analysis of 23 studies to review the effect of web-based lifestyle modification on weight control. They found that the internet component in obesity management had a modest but significant effect on weight control compared with non-use of the internet, and this effect especially seemed to depend on the type and time expended using Internet. Currently, several web-based weight loss programs have been developed and they are being tested to evaluate their usefulness in real-case scenarios [23].

Which web technology is the best design, and how its use could enhance the effectiveness of weight loss programs, remains unclear in most of the cases; improved software development by IT professionals and the appropriate use by health care providers are outcomes yet to improve [24].

\section{M-Health - Mobile phone and smartphone based tools}

Smartphone-based healthcare technology has undergone significant development over the last few years and is one of the most popular tools of e-Health. These devices are readily available and the designers of these devices envision the possibility of incorporating sensors [21] that can detect movement (via accelerometer), physical location (via the global positioning system and the presence of wireless networks), and other environmental and behavioral characteristics. There are concerns with data confidentiality, and healthcare providers determine that this is a significant issue that needs to be considered and resolved before widespread adoption by both patients and providers [25]. We also need to consider that the availability of new advanced software applications ("apps") for weight management grows every day but just a limited number of them have been scientifically tested by professionals. Studies have shown that mobile phone interventions are generally well accepted by patients and linked to an almost immediate weight loss and that advantages to using the telephone included overcoming transportation, distance, financial and time barriers [26].

Weinstrock et al. [27] designed the SHINE (Support, Health, Information, Nutrition and Exercise) program, a telephone intervention through individual visits (IC) or conference calls (CC) of DPP intensive lifestyle program. They showed a significant weight loss in patients with metabolic syndrome at 1 and 2 years and this effect was greater for CC than for IC group. Mean weight loss was $6.2 \mathrm{Kg}$ (CC) vs. $2.2 \mathrm{Kg}$ (IC) $(\mathrm{p}<0.001)$ at year 2 . The interaction between members created a supporting environment that helped with the goal losing weight and maintaining the weight loss. A text message based intervention also showed that the patients with overweight who received personalized SMS and MMS messages 2-5 times daily lost more weight and even would recommend the intervention to others [28].

In an effort to evaluate the feasibility, effectiveness and costeffectiveness of a telephone-based weight loss service and an existing face-to-face, group-based service, Whelan et al. [29] designed a nonrandomized, two-arm feasibility trial where patients who declined a two-month existing outpatient group-based program were offered a six-month research-based telephone program. The telephone program achieved significant weight loss $(-4.1 \pm 5.0 \%$; $\mathrm{p}=0.001)$ for completers 
( $\mathrm{n}=35 ; 57 \%$ of enrollees) at six months compared to the outpatient group-based program ( $\mathrm{n}=33$ completers; $66 \%,-2.0 \%[-3.4,-0.6]$; $\mathrm{p}=0.007)$ and the cost per healthy life year gained was $\$ 33,000$ and $\$ 85,000$ respectively. They concluded that telephone delivered weight management services could be successful and cost-effective within an acute-care hospital setting, likely more so than usual (group-based) care and it would help to overcome the difficulties of attending the face-to-face group based sessions offered during working hours.

Holzapfel et al. [30] also conducted a study to evaluate the effectiveness of a weight management program through regular phone calls providing individualized lifestyle recommendations, and delivery of printed materials to 258 participants. The mean weight change in the completers, was $-4.25 \mathrm{~kg} \pm 5.18 \mathrm{~kg}(\mathrm{p}<0.001)$, which corresponds to a mean percentage body weight change of $-4.10 \mathrm{~kg} \pm 4.88 \%$ after 12 months which may be comparable to face-to-face interventions.

Bacigalupo et al. [31] reviewed all published randomized controlled trials consisting of interventions using mobile technology for therapy of overweight and obesity. The interventions comprised of participants being educated about weight loss via diet and exercise and carrying with them in a mobile device (i.e. a text pager, mobile phone or other) their waking hours, that was used as a motivational behavior change tool. In some cases, the mobile device was a tool through which the participant received a motivational message, in others, it was used to record food intake and/or physical activity as well as daily energy balance. This review showed strong scientific evidence for short-term weight-loss and moderate evidence for medium-term weight-loss, through use of mobile technologies as part of intervention delivery [30].

Bhardwaj et al. [32] evaluated the efficacy and related costs of mobile interventions directed toward adult obesity in the US, in comparison to conventional care. Lifestyle intervention related to nutrition, physical activity and sedentary behavior were mainly addressed. They noticed that the effects of $\mathrm{m}$-health apps on waist circumference and BMI were generally positive, as were the results measuring changes in dietary behavior. They also noticed that the effects of obesity-related apps on patient's satisfaction and adherence were positive and cost-effective.

A recent study by Thomas et al. [33] (Health-E-Call) showed that smartphones could be a promising tool enhancing key components of behavioral weight loss treatments. Their study showed that patients who received behavioral weight loss treatment consisting of smartphonebased self-monitoring, feedback and behavioral skills training lost an average of $10.9 \mathrm{~kg}$ at 24 weeks.

Based on the scientific evidence currently available, mobile phones as a tool for weight loss, seem to have a greater effect on helping patient is to accomplish weight loss, although further research is necessary with larger samples in different populations and a longer-term follow up.

\section{Social media}

A recent review [34] of 42 articles studying the use of social media in chronic disease concluded that this tool could provide social, emotional and experimental support to different types of patients. Of the 42 studies reviewed, 9 included patients with obesity and in 6 of these 9 , the social media effect was considered positive ( 3 studies used blogs, 2 used Facebook and 1 used Twitter). These results suggest that the use of social media tools, especially Facebook and blogs, could help to improve clinical outcomes in weight management.

There is also the possibility of using virtual-reality video games to promote physical activity, group support and rewards, and physical activity monitors. Some of these new tools need to be evaluated in extended clini-cal trials to evaluate their real effect in weight management.

\section{Association of e-Health elements}

In general, most of the clinical trials about telemedicine and obesity incorporate various types of technology aiming to achieve the best outcomes in obesity management. In a review and metaanalysis executed by Hutchesson et al. [35] approximately $40 \%$ of the interventions were based on the association of several types of technologies and they concluded that e-Health interventions with extra features or components were more effective than standard programs. Adding monitoring devices or mobile applications to standard care achieved more weight loss than not including any e-Health element.

Appel et al. [36] conducted a clinical trial in 415 obese patients randomized in three groups; the first one received remote support by telephone calls, website and email; the second one added face to face support by individual and group sessions; and the last one was the selfdirected control group. At the end of 24 months, the mean change in weight from baseline was $-0.8,-4,6$ and $-5,1 \mathrm{~kg}(\mathrm{p}<0.001)$ in control, remote support and face to face support respectively. Castelnuovo et al. [37] in the TECNOB ("Technology for obesity"), a randomized controlled trial of a multidisciplinary telecare interventions (multi sensor armband, web-site, e-mail, chat lines, videoconference, telephone and mobile phone) for patients with obesity and type2 diabetes, during 12 months of follow up, did not show differences versus the conventional treatment; although, part of this observation may have been secondary to the low number of patients included in the project.

Luley et al. [38] carried out a randomized parallel group open trial with 3 groups of patients with metabolic syndrome: Control, ABC (Active Body Control Program of University of Magdeburg with information in weekly letters sent by mail or e-mail) and $4 \mathrm{~S}$ (sigma telephone coaching with monthly telephone calls). Partic-ipants were given accelerometers to investigate the efficacy of tele-monitoring physical activity and nutrition over 12 months. They showed that telemonitoring improved weight loss and markers of metabolic syndrome [37] after one year; however, during the follow-up period after the second year, significant weight regain occurred in both intervention groups [39].

Recent data reported [40] that 54\% of the technology-based interventions which promoted a healthy lifestyle helped patients with overweight to lose weight. They concluded that currently we lack scientific evidence regarding what would be the optimal use of technology in weight loss and advocated the need for future research, to better define the application of these technologies in patients with obesity.

The following are three groups where the necessity for more research is evident:

\section{e- HEALTH and Primary Care obesity management}

An increased reliance on universal technology availability makes Telemedicine a potentially viable option to accommodate visual, physical, hearing and cognitive impairments even in rural areas and for older adults; it minimizes patient travel time, missed work, costs and risks associated with travel. Patients can use existing home-based technologies such as personal computers or tablets and obtain medical care and counseling without the need of leaving their homes, reducing the risk for high risk elderly to fall, particular during the winter, and e-health may reduce the cost of associated travel and other indirect costs [41]. 


\section{e- HEALTH and childhood obesity}

ICTs might to be a useful tool for prevention and treatment of children and teenagers with obesity, The Hop-SCOTCH was a randomized controlled trial based on real-world implementation of web-based share care software conducted at the Royal Children's Hospital with 22 general practices (GPs) across Melbourne for obesity management of 3-10 years old children. The GPs felt that the software was useful but there were many technological barriers (e.g., outdated hardware, poor internet connections) that affected it is proper use [24]

Chen et al. [42] conducted a systematic review of the studies evaluating the efficacy of technology-based interventions for obesity preventions in adolescents (aged 12-18 years). The type of intervention consisted of Internet or active video games. The BMI decreased in 6 out of 14 studies evaluated; they noticed improve-ment of physical activity outcomes (in 6 out of 11) and in dietary behavior and psychological function ( 5 out of 7 studies). Based on these outcomes they concluded that there is no clear evidence that technology-based interventions decrease obesity in adolescents, in part due to the short-term follow up and the wide range of ages included. They also underscored the lack of evidence regarding the optimal dose or duration of the in-tervention (they estimated minimum 1 hour/weekly for 10-16 weeks to be effective) or the most effective type of technology (younger adolescents might prefer video games and interaction with peers, while older adolescents might prefer a smartphone app or Internet-based program).

Smith et al. [43] evaluated the effect of health information technology (9 involved electronic health records and computerized decision support, 2 used telemedicine and 3 used text message or telephone support) on outcomes and care processes in pediatric obesity management. The results demonstrated that Telemedicine counseling was associated with changes in BMI percentile similar to that of inperson counseling, improved treatment access in 2 studies, and that text messaging or telephone support was associated with weight loss maintenance in 1 of 3 studies. In childhood obesity, studies show promise for telemedicine to expand treat-ment access and for EHRs to assist physicians in adhering to clinical guidelines for screening. To date, the effect on weight loss and behavior change remains understudied and inconsistent.

\section{E- HEALTH and bariatric surgery}

Weight regain after bariatric surgery is a main concern and behavioral modification is a key factor for successful maintenance of weight loss long- term. It has been suggested that e-Health technologies could help patients get the best possible outcome after bariatric surgery. The use of accelerometers, palmtop computers, and mobile phones as tools to collect information about eating patterns, physical activity and feelings in real time, may be beneficial to encourage a healthy life style. In addition, some commercial programs that need to be evaluated have been designed specifically for bariatric surgery patients [44]. More studies are needed in this population to assess the most appropriate intervention.

There are some limitations present in the studies cited above, such as heterogeneous inclusion criteria, short-term follow up and high attrition rate. Most of the studies lasted less than 1 year, which is suboptimal, especially when studying a chronic disease such as obesity.

Lastly, an important point to keep in mind is reimbursement for distant health [45]. The reason why many physicians do not feel comfortable incorporating telemedicine care into their practice models it is due to confusion surrounding provider reimbursement for these visits. Currently, there is no set standard for private health insurance providers regarding telemedicine. Telemedicine reimbursement varies by location, services provided, and payers. Insurance companies are starting to recognize the value of telemedicine and many of them, accept this healthcare innovation and have been steadily broadening coverage. Medicare is the most focused on geographic restrictions, while many state Medicaid programs and private insurers are more likely to see telemedicine as location-agnostic. Many Medicare Advantage enrollees are already covered for tele-medicine services, regardless of location. Telemedicine in Medicaid is also covered in 48 states and each state has flexibility to determine how it will reimburse for this service. There are positive indicators for reimburse-ment across all three major payer types, but still in many cases a prior approval is needing before a telemedi-cine visit will be reimbursed.

\section{Conclusions}

Based on the studies reviewed, it seems useful to incorporate ICT in the prevention and treatment of obesity when taking into account its advantages. Scientific evidence has shown that it is a viable approach due to improved availability, self-monitoring by the patients, fast and easy access to care, group support models, scalability cost-effectiveness, and long-term follow-up opportunities.

The "gold standard" e-Health tool for weight management should be based on behavioral change therapy, self-monitoring of individual data, personalized recommendations and feedback, and, most importantly, cost effectiveness for the patient and the provider and our healthcare system in general.

At the same time we lack clear evidence, about which ICT intervention is the most efficacious; further investigation is required to determine the optimal type, effectiveness, duration, frequency, and proper type and frequency of contact with health professionals, in order to get the best cost-effectiveness when using e-Health elements to treat overweight and obesity.

Healthcare professionals need to take into account the fast development of new technologies and new apps, as these technologies are becoming more and more validated by technical and healthcare professionals for adequate patient care.

Finally, the use of e-Health, and especially telemedicine, in overweight and obesity treatment has the potential to be a very effective tool to help patients improve their lifestyle, and perhaps assist them in maintaining a healthy lifestyle change for the longterm. The use of e-Healthy may improve patient access to patients with obesity and facilitate exchange of clinical data. By sharing care between professionals such as primary care and specialties, healthcare professionals can minimize travel costs to families, promote earlier initiation of effective treatment, and yield a cost-effective approach to patient care. Ultimately, the goal is to have at least similar outcomes with e-Health compared to face to face obesity therapy.

\section{References}

1. Cullen KA, Mace KE, Arguin PM; Centers for Disease Control and Prevention (CDC) (2016) Malaria Surveillance - United States. MMWR Surveill Summ 65: 1-22. [Crossref]

2. World Health Organization Media Centre. http://www.who.int/mediacentre/factsheets/fs 31

3. Kelly T, Yang W, Chen CS, Reynolds K, He J (2008) Global burden of obesity in 2005 and projections to 2030. Int J Obes (Lond) 32: 1431-1437. [Crossref]

4. World Health Organization (2016) Global Action Plan for the Prevention and Control of NCDs 2013-2020.

5. Pagliari C, Sloan D, Gregor P, Sullivan F, Detmer D, et al. (2005) What is eHealth (4) a scoping exercise to map the field. J Med Internet Res 7: e9. [Crossref] 
6. Eysenbach G (2001) What is e-health? J Med Internet Res 3: E20. [Crossref]

7. Della Mea V (2001) What is e-Health (2): the death of telemedicine? J Med Internet Res 3: e22. [Crossref]

8. Silva BM, Rodrigues JJ, de la Torre Díez I, López-Coronado M, Saleem K (2015) Mobile-health: A review of current state in 2015. J Biomed Inform 56: 265-272. [Crossref]

9. Whitehead L, Seaton P (2016) The effectiveness of self-management mobile phone and tablet apps in long-term condition management: A systematic review. J Med Internet Res 18: e97. [Crossref]

10. Pew Research Center (2015) The Smartphone Difference.

11. Jensen MD, Ryan DH, Apovian CM, Ard JD, Comuzzie AG, et al. (2013) AHA/ACC/ TOS guideline for the management of over-weight and obesity in adults: a report of the American College of Cardiology/American Heart Association Task Force on Practice Guidelines and The Obesity Society. Circulation 129: S102-S138.

12. Look AHEAD Research Group (2014) Eight-year weight losses with an intensive lifestyle intervention: the look AHEAD study. Obesity (Silver Spring) 22: 5-13. [Crossref]

13. Knowler WC, Fowler SE, Hamman RF, et al. (2009) Diabetes Prevention Program Research Group. 10-year fol-low-up of diabetes incidence and weight loss in the Diabetes Prevention Program Outcomes Study. Lancet 374: 1677-1686.

14. Burguera B, Jesús Tur J, Escudero AJ, Alos M, Pagán A, et al. (2015) An Intensive Lifestyle Intervention Is an Effective Treatment of Morbid Obesity: The TRAMOMTANA Study-A Two-Year Randomized Controlled Clinical Trial. Int J Endocrinol 2015: 194696. [Crossref]

15. Montesi L, El Ghoch M, Brodosi L, Calugi S, Marchesini G, et al. (2016) Long-term weigth loss maintenance for obesity: a multidisciplinary approach. Diabetes Metab Syndr Obes 9: 37-46. [Crossref]

16. Rosser BA, Vowles KE, Keogh E, Eccleston C, Mountain GA (2009) Technologicallyassisted behavior change: a systematic review of studies of novel technologies for the management of chronic illness. J Telemed Telecare 15: 327-338. [Crossref]

17. Avery G, Cook D, Talens S (2016) The Impact of a Telephone-Based Chronic Disease Management Program on Medical Expenditures. Popul Health Manag 19: 3.

18. Wadden TA, Neiberg RH, Wing RR, Clark JM, Delahanty LM, et al. (2011) Four-year weight losses in the Look AHEAD study: factors associated with long-term success. Obesity (Silver Spring) 19: 1987-1998. [Crossref]

19. Gilmore LA, Duhé AF, Frost EA, Redman LM (2014) The technology boom: a new era in obesity management. J Diabetes Sci Technol 8: 596-608. [Crossref]

20. Burke LE, Wang J, Sevick MA (2011) Self-monitoring in weight loss: a systematic review of the literature. J Am Diet Assoc 111: 92-102. [Crossref]

21. Thomas JG, Bond DS (2014) Review if innovations in digital health technology to promote weight control. Curr Diab Rep 14: 485. [Crossref]

22. Kodama S, Saito K, Tanaka S, Horikawa C, Fujiwara K, Hirasawa R et al. (2012) Effect of Web-based lifestyle modification on weight control: a meta-analysis. Int J Obes (Lond) 36: 675-685. [Crossref]

23. Dennison L, Morrison L, Lloyd S, Phillips D, Stuart B, et al. (2014) Does Brief Telephone Support Improve Engagement With a Web-Based Weight Management Intervention? Randomized Controlled Trial. J Med Internet Res 16: e95. [Crossref]

24. Lycett K, Wittert G, Gunn J, Hutton C, Clifford SA, et al. (2014) The challenges of realworld implementa-tion of web-based shared care software: the HopSCOTCH SharedCare Obesity Trial in Children. BMC Med Inform Decis Mak 14: 61. [Crossref]

25. Tagliente I, Solvoll T, Trieste L, De Cecco CN, Murgia F, et al. (2016) Which indicators for measuring the dai-ly physical activity? An overview on the challenges and technology limits for Telehealth applications. Technol Health Care 24: 665-672. [Crossref]
26. Aguilar-Martínez A, Solé-Sedeño JM, Mancebo-Moreno G, Medina FX, CarrerasCollado R, et al. (2014) Use of mobile phones as a tool for weight loss: a systematic review. J Telemed Telecare 20: 339-349. [Crossref]

27. Weinstrock R, Trief P, Cibula D, et al. Weight Loss Success in Metabolic Syndrome by Telephone Inter-ventions: Results from the SHINE Study. J Gen Intern Med 28: 1620-1628. [Crossref]

28. Patrick K, Raab F, Adams MA, Dillon L, Zabinski M, et al. (2009) A text messagebased intervention for weight loss: randomized con-trolled trial. J Med Internet Res 11: e1. [Crossref]

29. Whelan ME, Goode AD, Eakin EG, Veerman JL, Winkler EA, et al. (2016) Feasibility, effec-tiveness and cost-effectiveness of a telephone-based weight loss program delivered via a hospital outpa-tient setting. Transl Behav Med 6: 386-395. [Crossref]

30. Holzapfel C, Merl M, Stecher L, Hauner H (2016) One-Year Weight Loss with a Telephone-Based Lifestyle Program. Obes Facts 9: 230-240. [Crossref]

31. Bacigalupo R, Cudd P, Littlewood C, Bissell C, Hawley M. S, Buckley Woods H. Interventions employ-ing mobile technology for overweight and obesity: an early systematic review of randomized controlled trials. Obesity reviews (2013) 14, 279 291. [Crossref]

32. Bhardwaj NN, Wodajo B, Gochipathala K, Paul DP 3rd, Coustasse A (2017) Can mHealth Revolutionize the Way We Manage Adult Obesity? Perspect Health Inf Manag 14: 1a. [Crossref]

33. Thomas JG, Wing RR (2013) Health-e-call, a smartphone-assisted behavioral obesity treatment: pilot study. JMIR Mhealth Uhealth 1: e3. [Crossref]

34. Patel R, Chang T, Greysen SR, Chopra V (2015) Social Media Use in Chronic Disease: A Systematic Review and Novel Taxonomy. Am J Med 128: 1335-1350. [Crossref]

35. Hutchesson MJ, Rollo ME, Krukowski R, Ells L, Harvey J, et al. (2015) eHealth interventions for the prevention and treatment of overweight and obesity in adults: a systematic review with meta-analysis. Obes Rev 16: 376-392. [Crossref]

36. Appel LJ, Clark JM, Yeh HC, Wang NY, Coughlin JW, et al. (2011) Comparative effec-tiveness of weight-loss interventions in clinical practice. N Engl J Med 365: 19591968. [Crossref]

37. Castelnuovo G, Manzoni GM, Cuzziol P, Cesa GL, Corti S, et al. (2011) TECNOB Study: Ad Interim Results of a Randomized Controlled Trial of a Multidisciplinary Telecare Intervention for Obese Patients with Type-2 Diabetes. Clin Pract Epidemiol Ment Health 7: 44-50. [Crossref]

38. Luley C, Blaik A, Gotz A, Kicherer F, Kropf S, et al. (2014) Weight loss by telemonitoring of nutrition and physical activity in pa-tients with metabolic syndrome for 1 year. J Am Coll Nutr 33: 363-374. [Crossref]

39. Stumm G, Blaik A, Kropf S, Westphal S, Hantke T, et al. (2016) Long-Term FollowUp of the Telemonitor-ing Weight-Reduction Program (Active Body Control). Journal of Diabe-tes Research 2016: 7.

40. Raaijmakers LC, Pouwels S, Berghuis KA, Nienhuijs SW (2015) Technology-based interventions in the treat-ment of overweight and obesity: A systematic review. Appetite 95: 138-151. [Crossref]

41. Batsis J, Pletcher S, Stahl J (2017) Telemedicine and primary care obesity managemen in rural areas -innovative approach for older adults? BMC Geriatrics 17: 6.

42. Chen JL, Wilkosz ME (2014) Efficacy of technology-based interventions for obesity prevention in adolescents: a systematic review. Adolesc Health Med Ther 5: 159-170. [Crossref]

43. Smith A, Skow A, Bodurtha J, Kinra S (2013) Health Information Technology in Screening and Treatment of Child Obesity: A Systematic Review. Pediatrics 131: e894-902. [Crossref]

44. Thomas JG, Bond DS, Sarwer DB, Wing RR (2011) Technology for behavioral assessment and intervention in bariatric surgery. Surg Obes Relat Dis 7: 548-557. [Crossref]

45. https://www.americanwell.com/telemedicine-reimbursement/

Copyright: (C2017 Dueñas S. This is an open-access article distributed under the terms of the Creative Commons Attribution License, which permits unrestricted use, distribution, and reproduction in any medium, provided the original author and source are credited. 